\title{
Structure-property relationship of nanocrystalline tin dioxide thin films grown on (1012) sapphire
}

\author{
X. Q. Pan, ${ }^{\text {a) }}$ L. Fu, and J. E. Dominguez \\ Department of Materials Science and Engineering, The University of Michigan, Ann Arbor, Michigan 48109
}

(Received 11 September 2000; accepted for publication 5 March 2001)

This work demonstrates the correlation between the microstructure of nanocrystalline $\mathrm{SnO}_{2}$ thin films and their electrical transport properties and sensitivities to reducing gases. $\mathrm{SnO}_{2}$ thin films were deposited on the (1012) surface of $\alpha-\mathrm{Al}_{2} \mathrm{O}_{3}$ (sapphire) using electron beam evaporation of a pure $\mathrm{SnO}_{2}$ ceramic source, followed by postdeposition annealing in synthetic air. $\mathrm{SnO}_{2}$ thin films with randomly oriented nanosized grains were obtained by annealing an amorphous $\mathrm{SnO}$ film deposited at room temperature. Films with nanosized $\mathrm{SnO}_{2}$ laminates were obtained by annealing epitaxial $\alpha$-SnO films deposited at $600^{\circ} \mathrm{C}$. The laminates are oriented with their (101) planes parallel to the substrate surface and have a high density of coherent twin boundaries. Hall measurements indicate that the electron concentration of the film with laminate grains is much lower than for the film with random grains. It is proposed that the high density twin boundaries inside the laminates trap conducting electrons and significantly reduce the electron concentration. As a result, the sensitivity to reducing gases of the laminar film is higher than that of the corresponding film with randomly oriented $\mathrm{SnO}_{2}$ grains. It was also found that the grain size has strong effects on the sensitivity of $\mathrm{SnO}_{2}$ films. (C) 2001 American Institute of Physics. [DOI: 10.1063/1.1368866]

\section{INTRODUCTION}

Owing to its high chemical and mechanical stability, $\mathrm{SnO}_{2}$ with the rutile type structure is extensively used in many applications such as solar cells, catalytic support material, transparent electrodes, and reducing gas sensors. ${ }^{1,2}$ $\mathrm{SnO}_{2}$ is widely used as a base material in gas alarm devices on domestic, commercial, and industrial premises due to its high sensitivity to small concentrations of gases (at ppm levels). In particular, $\mathrm{SnO}_{2}$ thin films have drawn much interest because of their potential application and integration with microsensor devices. ${ }^{3}$ Considerable attention has recently been focused on the development of solid state gas sensors based on thin films with a crystallite size smaller than the Debye length of the material, which show increased gas sensitivity and short response time. ${ }^{4,5}$ Taking into account its potential applications and importance for fundamental research as well as its simple structure, (rutile-type) $\mathrm{SnO}_{2}$ is an ideal model system for a systematic investigation of the microstructure effects on physical and chemical properties.

Solid state oxide semiconductor gas sensors undergo changes in their conductance (or capacitance) during their interaction with certain molecules in the gas phase. When an oxide thin film is exposed to an oxygen atmosphere, oxygen adsorbs on the surface and traps free electrons from the oxide. These chemical reaction processes result in the formation of an electron depletion layer near the oxide surface. The formation of this depletion layer creates a Schottky barrier that controls the transport properties of free electrical carriers in the film. ${ }^{6-8}$ When reducing gaseous molecules are introduced into the oxygen atmosphere, they react with the

${ }^{a)}$ Electronic mail: panx@umich.edu adsorbed oxygen and desorb from the surface, decreasing the oxygen coverage. As a result, the Schottky barrier height can be modulated by the introduction of reducing gases, which causes a change in the conductance of the sensing element accordingly. The characteristics of the surface barrier strongly depend upon the concentration and mobility of electrical carriers existing in the oxide thin films. Furthermore, the concentration and mobility of conducting electrons of oxide thin films strongly depend on the microstructure and crystal defects. ${ }^{9,10}$ For instance, grain boundaries in an $n$-type oxide semiconductor such as $\mathrm{SnO}_{2}$ can trap electrons and form back-to-back Schottky barriers. In a nanocrystalline thin film, the width of grain boundary barriers becomes comparable with the grain size, and the electrical transport phenomena can be quite different from that found in the bulk material. Furthermore, the electrical carrier concentration of oxide significantly depends on the stoichiometry of the material. The electrical carriers in $\mathrm{SnO}_{2}$ mainly originate from the oxygen vacancy donors, and the electrical properties are strongly controlled by chemical composition. Evidence also shows the strong dependence of optical properties on the microstructure, crystal defects, and chemical composition. The latter are strongly influenced by the processing conditions, which include deposition temperature, substrate structure, and postdeposition annealing.

It has been reported that deposition temperatures ${ }^{11}$ and annealing conditions ${ }^{12}$ have strong influences on the properties of $\mathrm{SnO}_{2}$ thin films synthesized by electron-beam evaporation followed by annealing in air. Reddy et al. ${ }^{13}$ found that films deposited at 25 and $350^{\circ} \mathrm{C}$ are more suitable for sensor application compared to films deposited at intermediate temperatures and heat treated under identical conditions. Cho et al. ${ }^{14}$ reported the effects of annealing on the electrical 
properties of $\mathrm{SnO}_{2}$ films prepared by electron beam evaporation. They found that the resistance of the $\mathrm{SnO}_{2}$ thin films changed as a function of annealing temperature and reached the minimum at about $327 \mathrm{~K}$. Most of the studies reported so far on tin oxide films fabricated by the electron-beam evaporation method focused on the influences of preparation conditions on electrical and optical properties of these films. However, there is little work on microstructure characterization, which is key to understanding the physical properties of the films. Hence a systematic study of the microstructure of $\mathrm{SnO}_{2}$ thin films is necessary to obtain a fundamental understanding of the structure-property relationships.

In this article we report our studies on nanocrystalline $\mathrm{SnO}_{2}$ thin films fabricated by electron beam evaporation of a pure $\mathrm{SnO}_{2}$ ceramic source followed by postdeposition annealing in synthetic air. The microstructure of thin films with different deposition and annealing conditions were studied by x-ray diffraction (XRD), scanning electron microscopy (SEM), and transmission electron microscopy (TEM). Gas sensing performance and electrical properties were determined by electrical measurements using four point probes with several gaseous environments. The correlation between microstructure and properties has been established based on the experimental results.

\section{EXPERIMENTAL METHODS}

$\mathrm{SnO}_{2}$ thin films were fabricated using electron beam evaporation of a $\mathrm{SnO}_{2}$ ceramic source material. Disks of $\mathrm{SnO}_{2}$ ceramic were prepared by sintering a high purity $\mathrm{SnO}_{2}$ powder synthesized by the chemical coprecipitation method. The substrate material used in this study was single crystal $\alpha-\mathrm{Al}_{2} \mathrm{O}_{3}$ (sapphire) with a (1012) surface orientation (Rcut). The detailed description of the experimental procedure was given in previous works. ${ }^{15,16}$

The microstructure of $\mathrm{SnO}_{2}$ thin films was characterized by a combination of XRD and TEM. The surface morphology of the films was studied by SEM. Cross-section TEM specimens were prepared by a standard procedure, which includes mechanical grinding; polishing, precision dimpling, and ion milling. The final thinning of specimens was carried out on a Gatan precision ion polishing system (PIPSTM, Model 691) using an accelerating voltage of $4.5 \mathrm{kV}$ and an incident angle of $4^{\circ}-6^{\circ}$.

The electrical properties of thin films were determined by Hall effect measurements in a $2.5 \mathrm{kG}$ magnetic field, using the van der Pauw method. The thin film samples were loaded into a gas reaction chamber. The gas flow and composition were controlled by a mass-flow-controller (MFC) (MKS Instruments, Andover, MA). The gases used were prepurified $\mathrm{N}_{2}(99.998 \%)$, extra dry $\mathrm{O}_{2}(99.6 \%)$, and diluted high purity reducing gases (typically $5 \%$ gas balanced in $\mathrm{N}_{2}$ ). Samples could be heated up to about $700^{\circ} \mathrm{C}$ with the temperature fluctuation of about $\pm 0.1^{\circ} \mathrm{C}$.

\section{RESULTS}

By the electron beam evaporation of a pure $\mathrm{SnO}_{2}$ ceramic source amorphous films were obtained when deposited at temperatures below $300{ }^{\circ} \mathrm{C}$ whereas epitaxial $\alpha$-SnO films
TABLE I. Deposition and annealing conditions of $\mathrm{SnO}_{2}$ films by electronbeam evaporation.

\begin{tabular}{cccc}
\hline \hline Sample & $\begin{array}{c}\text { Deposition } \\
\text { temperature }\left({ }^{\circ} \mathrm{C}\right)\end{array}$ & $\begin{array}{c}\text { Annealing } \\
\text { temperature }\left({ }^{\circ} \mathrm{C}\right)\end{array}$ & $\begin{array}{c}\text { Annealing time } \\
\text { (hours) }\end{array}$ \\
\hline A1-RT & $\sim 25$ & 700 & 2 \\
A2-RT & $\sim 25$ & 700 & 150 \\
A1-600 & 600 & 700 & 2 \\
A2-600 & 600 & 700 & 150 \\
\hline \hline
\end{tabular}

on the R-cut sapphire substrate were formed when deposited at $600{ }^{\circ} \mathrm{C} .{ }^{15}$ A postdeposition annealing in an oxygen atmosphere is necessary to transform the structure of the asdeposited films into the rutile-type $\mathrm{SnO}_{2}$ phase, which is desired for gas sensor application. Our previous studies suggest that the $\mathrm{SnO}$ film will fully transform into the rutile $\mathrm{SnO}_{2}$ phase by annealing at temperatures above $600{ }^{\circ} \mathrm{C}^{16}$ In this work, all as-deposited thin films were annealed at $700{ }^{\circ} \mathrm{C}$ for 2 or $150 \mathrm{~h}$ in flowing synthetic dry air $\left(0.8 \mathrm{~N}_{2} / 0.2 \mathrm{O}_{2}\right)$. The deposition and annealing conditions for all samples studied are summarized in Table I. Samples A1-RT and A2-RT were cut from the same piece of an as-grown amorphous film $(\mathrm{SnO})$ which was deposited at room temperature $\left(\sim 25^{\circ} \mathrm{C}\right)$. Samples A1-600 and A2-600 were cut from an epitaxial $\alpha$-SnO film grown at $600^{\circ} \mathrm{C}$. All films were annealed under identical conditions except for the annealing time, as shown in Table I.

Figure 1 shows the result of XRD $\theta-2 \theta$ scans for samples A1-RT and A1-600. All reflection peaks from these two samples are identified from either the rutile $\mathrm{SnO}_{2}$ structure or the sapphire $\left(\alpha-\mathrm{Al}_{2} \mathrm{O}_{3}\right)$ substrates. The XRD spectrum of sample A1-RT is similar to that of $\mathrm{SnO}_{2}$ powder, which indicates that the film is polycrystalline, with ran-
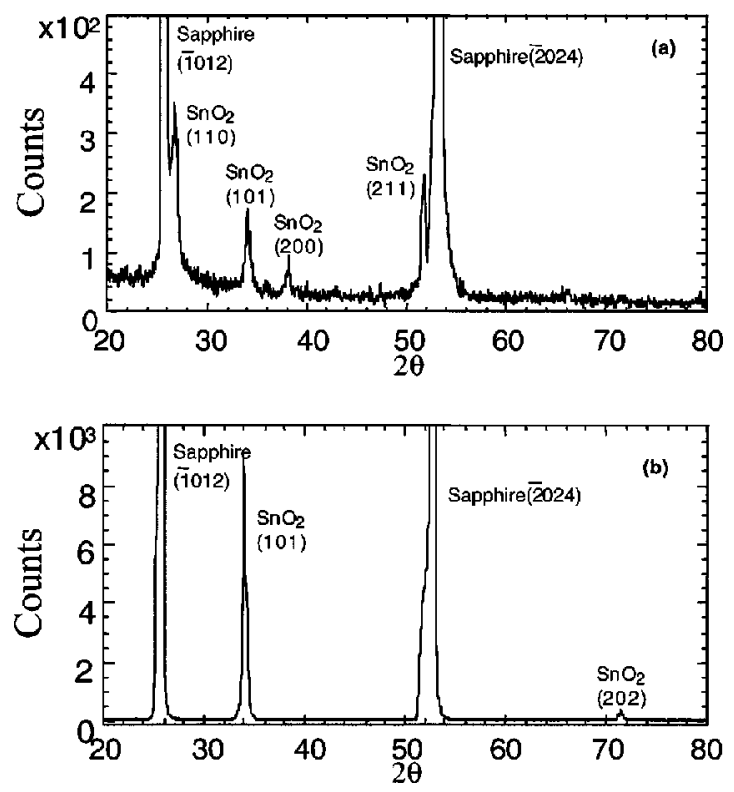

FIG. 1. X-ray diffraction patterns $(\theta-2 \theta)$ of $\mathrm{SnO}_{2}$ thin films obtained by annealing (a) an amorphous $\mathrm{SnO}$ film (A1-RT) and (b) an epitaxial $\alpha$-SnO film (A1-600) in air at $700{ }^{\circ} \mathrm{C}$ for $2 \mathrm{~h}$. The XRD pattern in (a) is similar to that of $\mathrm{SnO}_{2}$ powder, which indicates that the film has randomly oriented grains. The pattern in (b) only shows 101 reflection of $\mathrm{SnO}_{2}$. 

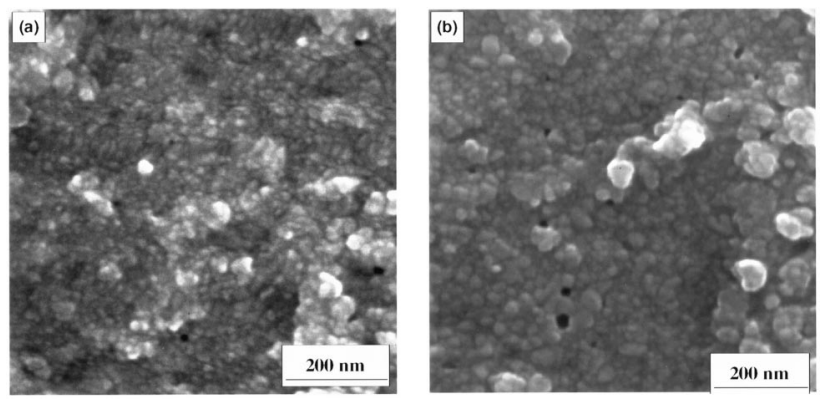

FIG. 2. SEM images showing the surface morphology of two $\mathrm{SnO}_{2}$ films, A1-RT and A2-RT, which were deposited at room temperature, followed by annealing in air for $2 \mathrm{~h}$ (a) and $150 \mathrm{~h}$ (b), respectively.

domly oriented grains. The XRD spectrum of sample A1-600 shows a reflection located at $33.9^{\circ}$, identified to be the 101 reflection of $\mathrm{SnO}_{2}$. Other reflections correspond to the substrate. This result indicates that an out-of-plane texture exists in the A1-600 $\mathrm{SnO}_{2}$ film. Moreover, the width of the diffraction peaks in sample A1-600 are much smaller than those in sample A1-RT, implying relatively large crystallites in the former film. The XRD results for sample A2-RT are similar to the one shown in Fig. 1(a). A2-600 shows the same diffraction pattern as A1-600.

Figure 2 shows SEM images of the surface morphology of the films deposited at room temperature and annealed for 2 and $150 \mathrm{~h}, \mathrm{~A} 1-\mathrm{RT}$ and A2-RT, respectively. Film A1-RT consists of very small grains with a mean size of $\sim 15 \mathrm{~nm}$. Some grains are connected to each other and form agglomerates. Film A2-RT has similar surface morphology as film A1-RT, however, its average grain size increases to $23 \mathrm{~nm}$ due to the long annealing time $(150 \mathrm{~h})$. Figure 3 shows SEM images of the surface morphology of the two $\mathrm{SnO}_{2}$ films (A1-600 and A2-600) deposited at $600{ }^{\circ} \mathrm{C}$ and annealed for 2 and $150 \mathrm{~h}$, respectively. The grain sizes in both films are distributed in a wide range between 20 and $300 \mathrm{~nm}$ and the average size is about $80 \mathrm{~nm}$. Through our SEM observation, pores with a size of $\sim 30 \mathrm{~nm}$ were found in all films.

Cross-section TEM investigations were carried out for samples A1-RT and A1-600. Figure 4(a) shows a crosssection micrograph taken from A1-RT which was obtained by annealing an amorphous tin oxide film at $700{ }^{\circ} \mathrm{C}$ for $2 \mathrm{~h}$. The film consists of both small, randomly shaped grains and columnar grains as seen in the middle of Fig. 4(a). The mean
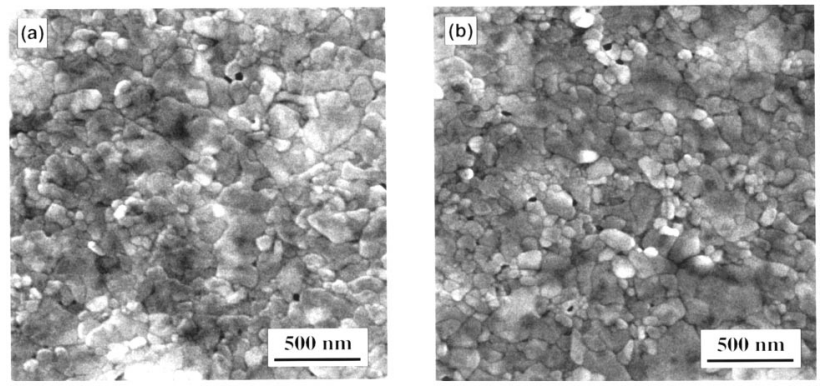

FIG. 3. SEM images showing the surface morphology of two $\mathrm{SnO}_{2}$ films, A1-600 and A2-600, which were deposited at $600{ }^{\circ} \mathrm{C}$, followed by annealing in air for $2 \mathrm{~h}$ (a) and $150 \mathrm{~h}(\mathrm{~b})$, respectively.
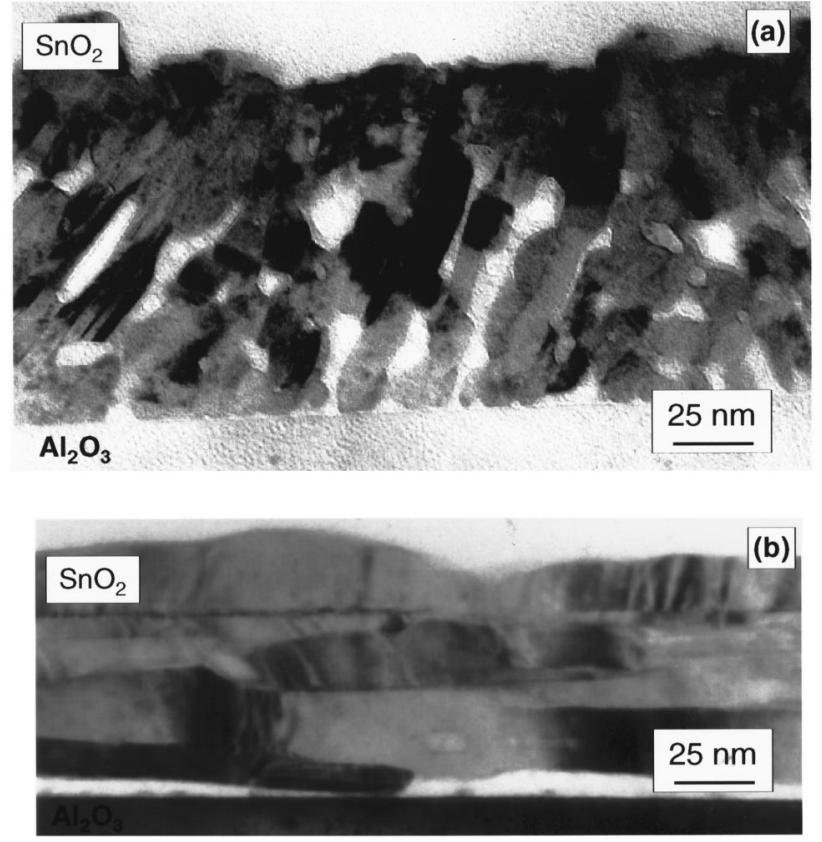

FIG. 4. Cross-section TEM images taken (a) from A1-RT which was obtained by annealing an amorphous tin oxide film at $700{ }^{\circ} \mathrm{C}$ for $2 \mathrm{~h}$ and (b) from A1-600 obtained by annealing the epitaxial $\alpha$-SnO film at $700{ }^{\circ} \mathrm{C}$ for $2 \mathrm{~h}$.

grain size in this film is $\sim 15 \mathrm{~nm}$, in agreement with the SEM measurements. Figure 4(b) is a cross-section TEM micrograph showing the microstructure of film A1-600, obtained by annealing the epitaxial $\mathrm{SnO}$ film at $700{ }^{\circ} \mathrm{C}$ for $2 \mathrm{~h}$. It is clearly seen in this image that the film consists of laminar grains. The laminas have an average thickness of $\sim 20 \mathrm{~nm}$. The grain size in lateral direction is $\sim 100 \mathrm{~nm}$. TEM studies were also conducted on film A2-600 that was obtained by annealing the same epitaxial $\mathrm{SnO}$ film at the same temperature as A1-600, but for $150 \mathrm{~h}$. It was found that the laminas in this film have a crystallite dimension along the lateral direction similar to that of A1-600, however, it has a larger average thickness of $28 \mathrm{~nm}$, compared to $20 \mathrm{~nm}$ in A1-600. This indicates that long time annealing results in the grain growth along the direction perpendicular to the laminar plane. According to TEM studies, we found that both A1600 and A2-600 films have a relatively low density with a mean pore size of $\sim 30 \mathrm{~nm}$. The existence of pores is probably due to the density difference between the as-grown epitaxial $\alpha$-SnO films and the final $\mathrm{SnO}_{2}$ films. The density of $\alpha$-SnO is $6.45 \mathrm{~g} / \mathrm{cm}^{3}$, while the rutile $\mathrm{SnO}_{2}$ phase has a density of $6.95 \mathrm{~g} / \mathrm{cm}^{3}$. Thus annealing causes a $7 \%$ volume shrinkage, which may result in the formation of pores in the film. Moreover, the three-dimensional growth of the rutile $\mathrm{SnO}_{2}$ grains will also lead to a rough surface and cause the formation of pores in the film. Another feature of the microstructure is that the $\mathrm{SnO}_{2}$ films (A1-600 and A2-600) obtained by annealing the epitaxial $\alpha$-SnO films consist of a high density of crystallographic shear planes and coherent $\{101\}$ twin boundaries parallel to the film/substrate interface. A typical example of such defect structures of a $\mathrm{SnO}_{2}$ laminate in A1-600 is shown in Fig. 5 of the previous paper. ${ }^{16}$ The formation of the twin boundaries is due to the phase 


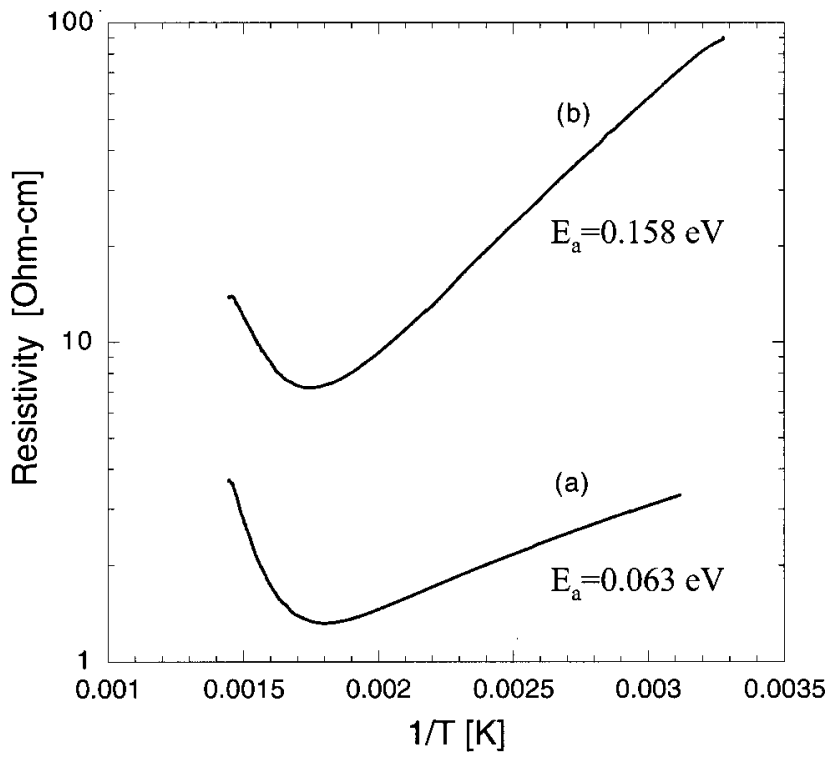

FIG. 5. Resistivity as a function of temperature for A1-RT (a) and A1-600 (b). Note that A1-600 has a laminar microstructure while A1-RT has randomly oriented nanosized grains.

transformation from the epitaxial $\alpha$-SnO to the rutile $\mathrm{SnO}_{2}$ phase during annealing in air. The detailed studies of the formation mechanisms are described in previous work.

The significant difference found between the microstructure (surface morphology, grain size, and shape) of the $\mathrm{SnO}_{2}$ thin films can influence the electrical transport properties of these films. Figure 5 shows the resistivity as a function of inverse temperature for A1-RT and A1-600. Figure 5(a) was obtained from A1-RT, whereas Fig. 5(b) was measured from A1-600. The activation energy determined from the slope of the resistivity-temperature curve is $0.063 \mathrm{eV}$ for A1-RT and $0.158 \mathrm{eV}$ for A1-600. These studies show that sample A1600 has higher resistivity and higher activation energy than sample A1-RT. Furthermore, Hall measurements revealed that the electrical transport properties of these samples are different. The electron concentration, Hall mobility, and electrical resistivity for each film, measured at room temperature, are summarized in Table II. The Hall mobility of conducting electrons is similar for both films. However, the conducting electron concentration of the sample A1-RT is one order of magnitude higher than that of A1-600. This is consistent with the high activation energy $(0.158 \mathrm{eV})$ of electrons and lower conductivity of sample A1-600. We would like to point out that the observed increase in resistivity with increasing temperature at about $350{ }^{\circ} \mathrm{C}$ is due to a decrease in electron mobility (thermal scattering) at high

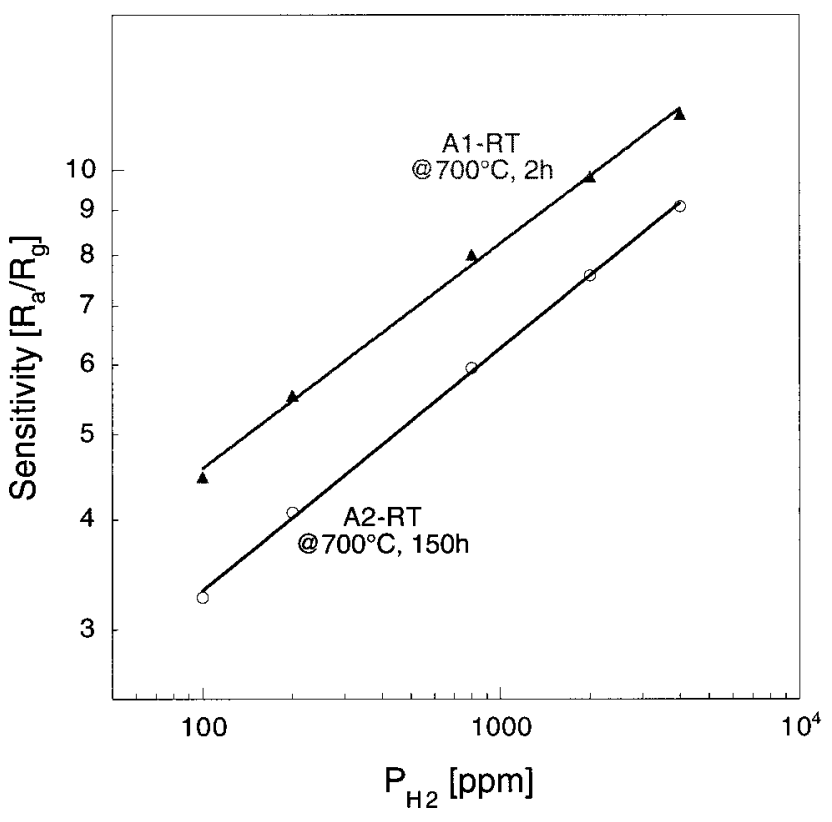

FIG. 6. Ratio of the resistance measured in synthesized air and that measured in $\mathrm{H}_{2}$ at $350{ }^{\circ} \mathrm{C}$ (sensitivity) for A1-RT (a) and A2-RT (b). Note the mean grain size is $15 \mathrm{~nm}$ for A1-RT and $23 \mathrm{~nm}$ for A2-RT.

temperatures. ${ }^{17}$ In addition, at these high temperatures oxygen reacts with the film and reduces the number of oxygen vacancies, ${ }^{18}$ thus decreasing the number of electrical carriers.

We have also evaluated the sensitivity of $\mathrm{SnO}_{2}$ films to reducing gases, which is defined as the resistance ratio $S$ $=R_{a} / R_{g}$, where $R_{a}$ and $R_{g}$ are the resistance measured in synthesized air and reducing gaseous environments, respectively. It was found that the $\mathrm{SnO}_{2}$ films studied in this work have the highest sensitivity when operated at $350{ }^{\circ} \mathrm{C} .{ }^{19}$ Figure 6 shows the response of A1-RT and A2-RT to $\mathrm{H}_{2}$ at $350^{\circ} \mathrm{C}$. The film annealed for $2 \mathrm{~h}(\mathrm{~A} 1-\mathrm{RT})$ has higher sensitivity than the film which was annealed for $150 \mathrm{~h}$ (A2-RT). This means that $\mathrm{SnO}_{2}$ film with smaller grain size has higher sensitivity. Figure 7 shows the response of A1-RT and A1600 to $\mathrm{H}_{2}$ at $350^{\circ} \mathrm{C}$. Figure 7 shows that the film A1-600 has higher sensitivity to $\mathrm{H}_{2}$ than A1-RT. These results indicate that the $\mathrm{SnO}_{2}$ film that consists of nanosized laminar grains with the (101) texture has better sensing performance to $\mathrm{H}_{2}$ than those obtained by annealing amorphous $\mathrm{SnO}$ film under the same annealing conditions, although the latter one has smaller grain size than the former one.

\section{DISCUSSION}

Tin dioxide is a wide band-gap $n$-type semiconductor. Its electrical transport behavior is controlled by the stoichiom-

TABLE II. Electrical transport properties of $\mathrm{SnO}_{2}$ films (A1-RT and A1-600) measured at room temperature.

\begin{tabular}{ccccc}
\hline \hline Sample & Microstructure & $n\left(\mathrm{~cm}^{-3}\right) \times 10^{16}$ & $\mu\left(\mathrm{cm}^{2} / \mathrm{V} \mathrm{s}\right)$ & $\rho(\Omega \mathrm{cm})$ \\
\hline A1-RT & $\begin{array}{c}\text { Random grains with an average } \\
\text { size of about } 15 \mathrm{~nm}\end{array}$ & 11.5 & 8.41 & 6.5 \\
A1-600 & $\begin{array}{l}\text { Laminar grains with (101) } \\
\text { texture; 80-90 nm in lateral } \\
\text { direction and 20 nm in } \\
\text { perpendicular direction }\end{array}$ & 1.1 & 9.12 & 62.5 \\
& & & \\
\hline \hline
\end{tabular}




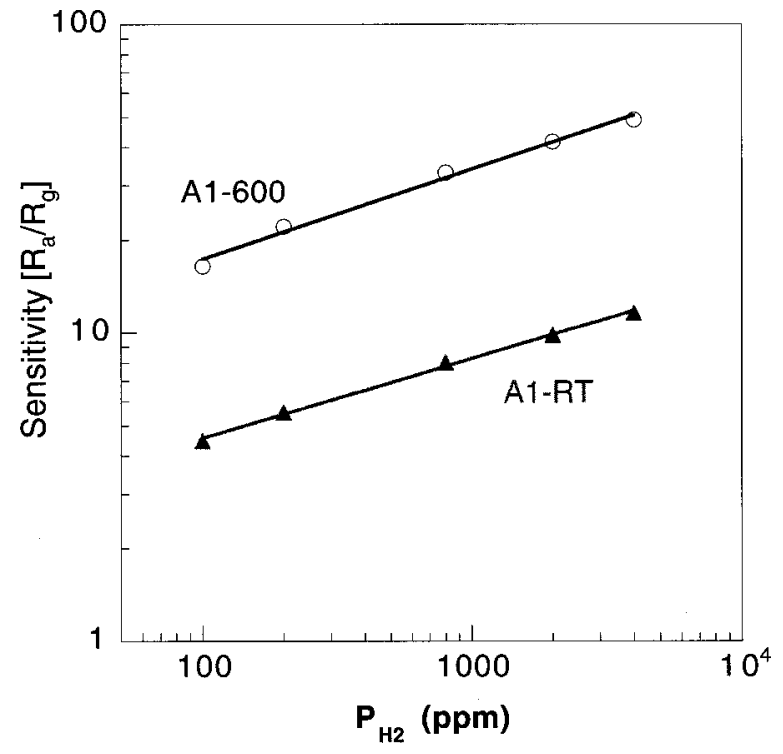

FIG. 7. Sensitivity to $\mathrm{H}_{2}$ of two $\mathrm{SnO}_{2}$ films (A1-RT and A1-600). Note that A1-600 has a laminar microstructure while A1-RT has randomly oriented nanosized grains.

etry and the microstructure of the material. The electrical conduction carriers (free electrons) mainly originated from the oxygen vacancy donors in the material. The effect of grain size and conductivity on the gas sensing properties of the films can be explained by taking into account the physical phenomena occurring during oxygen adsorption. Adsorbed oxygen species on the $\mathrm{SnO}_{2}$ grain surface traps electrons from the oxide in the near surface region and these electrons will not contribute to the electrical conductivity. As a result, an electron depleted layer forms near each $\mathrm{SnO}_{2}$ grain surface and builds up a potential barrier at the grain boundaries. For a given electrical carrier concentration in the material, a thicker depletion layer means that more electrons are extracted from the bulk and trapped on the grains surface. The depth of the electron depletion layer is characterized by the Debye length $L_{D}$, which is determined by the bulk donor level electron concentration in the material through the following equation

$$
L_{D}=\sqrt{\frac{\varepsilon_{s} k T}{q^{2} N_{D}}},
$$

where $\varepsilon_{s}$ is semiconductor permitivity, $k$ is the Boltzmann constant, $T$ is the temperature in Kelvin, $q$ is the electron charge, and $N_{D}$ is the donor concentration. The depletion layer needs to extend deeper in a material with low donor density in bulk, so that it could have enough electrons to ionize surface oxygen. The sensitivity $\left(R_{a} / R_{g}\right)$ is determined by the change of conductivity on exposure to reducing gases. The change in conductivity by the introduction of a reducing gas is greater for a film with lower electron concentration. Thus the more the film is depleted and also the closer $L_{D}$ is to the characteristic length of the system (thickness or grain size), the higher the gas sensitivity. Thus for a given electron concentration, an oxide film consisting of smaller grains has a higher sensitivity. This explains the difference in gas sensing performance of two $\mathrm{SnO}_{2}$ films (A1-RT and A2RT), which have similar electrical properties (electron con- centration and mobility) and microstructure except for the difference in grain size. The film A1-RT with a mean grain size of $15 \mathrm{~nm}$ shows a slightly higher sensitivity to $\mathrm{H}_{2}$ than the film A2-RT which has a mean grain size of $23 \mathrm{~nm}$.

Films with very different morphology, growth mechanism, and orientation also show very different electrical properties. Our experimental results showed that the electrical conductivity of the film A1-RT is higher than that of A1-600 (Fig. 5). The main differences in the microstructures between these two films are the grain size and grain orientation, as shown in Fig. 4. SEM studies (Figs. 2 and 3) showed that the film deposited at $600^{\circ} \mathrm{C}$ has a higher porosity than the one deposited at room temperature. The presence of pores in the film can create a discontinuous path for electron conduction and leads to the existence of neck conduction. This type of feature is known ${ }^{20}$ to decrease substantially the conductivity of the film by creating very inhomogeneous conduction (low resistance percolation paths) which is very difficult to measure using the Hall effect.

Hall measurements revealed that the electron carrier concentration in the film with laminar microstructure (A1600 ) is one magnitude lower than that in the film with random fine grain microstructure (A1-RT). On the other hand, the electron mobility is slightly greater for the film with a laminar structure. The mobility for polycrystalline films $(\mu)$, with a mean free path greater than the grain size, has been modeled as ${ }^{21}$

$$
\mu=\mu_{s}\left[l_{1} / l_{2}\right] C \exp (-\Delta E / k T),
$$

where $\mu_{s}$ is the single crystal mobility, $l_{1}$ is the grain size, $l_{2}$ the boundary width, $C$ a constant for a given material, and $\Delta E$ the grain boundary energy barrier. From the equation it can be observed that a greater grain size or smaller grain boundary width will result in a higher mobility. Figures 4(a) and 4(b) show that the laminar film has a greater grain size than the random film. It also shows that there are less grain boundary barriers parallel to electron conduction than in the random film. These two facts explain the greater mobility of the laminar film. However, this does not explain the lower electron concentration in the laminar film. Thus other mechanisms should be responsible for this difference. As shown in Fig. 5 of the previous article ${ }^{16}$ the films grown from epitaxial $\mathrm{SnO}$ (grown at $600^{\circ} \mathrm{C}$ ) have a high density of defects. The most abundant defects in the system are coherent (101) twin boundaries. These boundaries, due to their special crystallographic characteristics, do not contribute greatly to electron scattering. However, these twin boundaries cause the interruption of crystal lattice and trap conducting electrons, which creates an electrical barrier at the boundary. As a result, the existence of a large of number of twin boundaries can greatly lower the free electron concentration in the film and decrease the film conductivity, as shown in Fig. 5. Since films with laminar microstructure have low electron concentration, the depletion region is wider in these films and their sensitivity is higher than for the random grained films, as shown in Fig. 7.

The sensitivity can also be influenced by the grain orientation. Rohrer and coworkers ${ }^{22,23}$ investigated the orientation dependence of photochemical reactions on a rutile type 
$\mathrm{TiO}_{2}$ surface. They found that the most reactive surfaces in rutile $\mathrm{TiO}_{2}$ are near the $\{101\}$ orientation because of the fourcoordinate $\mathrm{Ti}$ on the $\{101\}$ surface. It was also reported that surfaces containing four-coordinate Ti had an increased selectivity for certain partial oxidation reactions. ${ }^{24}$ According to these results and the fact that $\mathrm{SnO}_{2}$ has the same rutile structure as $\mathrm{TiO}_{2}$, high sensivity may be expected for the $\mathrm{SnO}_{2}$ film which consists of $\{101\}$ oriented grains. This is consistent with our experimental results shown in Fig. 7. Also, it is possible that the $\{101\}$ oriented laminar grains chemisorbed more oxygen than the random grains during annealing, due to their higher reactivity. This chemisorbed oxygen (from annealing) would stay on the surface after annealing (strong chemical bond) and trap conduction electrons decreasing the conductivity of the laminar film with respect to the random film

\section{CONCLUSION}

In conclusion, it has been demonstrated that the electrical transport properties and the sensitivities to reducing gases of $\mathrm{SnO}_{2}$ thin films depend strongly upon the feature of microstructure and crystal defects. By controlling the deposition temperature during electron beam evaporation, nanocrystalline $\mathrm{SnO}_{2}$ thin films with either randomly oriented or laminar grains were produced after annealing. The film with laminar grains showed lower electron concentration and higher sensitivity to reducing gases. This was attributed to the great number of twin boundaries within the laminar grains, which acted as free electron trapping sites. For films with the same grain morphology, the grain size can be controlled by annealing temperature and time. The smaller the grain size the higher the sensitivity to reducing gases, due to an increased fraction of electron depletion in the smaller grains.

\section{ACKNOWLEDGMENTS}

The authors gratefully acknowledge the financial support of the National Science Foundation DMR 9875405 (CAREER-XQP), the Petroleum Research Fund No. 34093G5, the College of Engineering at the University of Michigan, and the Applied Materials Company (Santa Clara, CA).

${ }^{1}$ W. Göpel, J. Hesse and J. N. Zemel Chemical and Biochemical Sensors (VCH Weinheim, New York, 1991), Vol. 2.

${ }^{2}$ K. Ihokura and J. Watson, The Stannic Oxide Gas Sensor-Principles and Applications (CRC, Boca, Raton, FL, 1994).

${ }^{3}$ G. Advani and A. Jordan, J. Electrochem. Soc. 123, 29 (1990).

${ }^{4}$ N. Yamazoe, Proceedings of the Third International Meeting Chem. Sensors, Cleveland, OH, 1990, pp. 3-8.

${ }^{5}$ C. Xu, J. Tamaki, M. Miur, and N. Yamazoe, Sens. Actuators B 3, 147 (1991).

${ }^{6}$ H. Windischmann and P. Mark, J. Electrochem. Soc. 126, 627 (1979).

${ }^{7}$ P. K. Clifford and D. T. Tuma, Sens. Actuators 3, 233 (1983).

${ }^{8}$ S. R. Morrison, Sens. Actuators 11, 283 (1987).

${ }^{9}$ C. Xu, J. Tamaki, N. Miura, and N. Yamazoe, Sens. Actuators B 3, 147 (1991).

${ }^{10}$ V. Demarne and A. Grisel, Sens. Actuators B 7, 704 (1992).

${ }^{11}$ P. Tischer, H. Pink, and L. Treilinger, Jpn. J. Appl. Phys., Part 1 19, 513 (1989)

${ }^{12}$ M. H. Madhusudhana Reddy, S. R. Jawalekar, and A. N. Chandorkar, Thin Solid Films 169, 117 (1989).

${ }^{13}$ M. H. Madhusudhana Reddy and A. N. Chandorkar, Sens. Actuators B 9 , 1 (1992).

${ }^{14}$ W. I. Cho, H. Jang, and S. R. Lee, Scr. Metall. Mater. 32, 815 (1995).

${ }^{15} \mathrm{~L}$. Fu and X. Q. Pan, J. Electroceram. (submitted).

${ }^{16}$ X. Q. Pan and L. Fu, J. Appl. Phys. 89, 6048 (2001).

${ }^{17}$ C. G. Fonstad and R. H. Rediker, J. Appl. Phys. 42, 2918 (1971).

${ }^{18}$ S. Samson and C. G. Fonstad, J. Appl. Phys. 44, 4618 (1973).

${ }^{19}$ L. Fu, Ph.D. thesis, The University of Michigan-Ann Arbor, 2000

${ }^{20}$ H. Ogawa, M. Nishikawa, and A. Abe, J. Appl. Phys. 53, 4448 (1982).

${ }^{21}$ L. L. Kazmerski, in Polycrystalline and Amorphous Thin Films and Devices, edited by L. L. Kazmerski (Academic, New York, 1980), p. 90.

${ }^{22}$ P. A. Morris Hotsenpiller, J. D. Bolt, W. E. Farneth, J. B. Lowekamp, and G. S. Rohrer, J. Phys. Chem. B 102, 3216 (1998).

${ }^{23}$ J. B. Lowekamp, G. S. Rohrer, P. A. Morris Hotsenpiller, J. D. Bolt, and W. E. Farneth, J. Phys. Chem. B 102, 7323 (1998).

${ }^{24}$ K. S. Kim and M. A. Barteau, Surf. Sci. 223, 13 (1989). 